\title{
Prolonged interleukin-6 administration enhances glucose tolerance and increases skeletal muscle PPAR $\alpha$ and UCP2 expression in rats
}

\author{
Anna G Holmes ${ }^{1, *}$, Jose L Mesa ${ }^{1, *}$, Bronwyn A Neill ${ }^{1,2}$, Jason Chung ${ }^{1}$, Andrew L Carey ${ }^{2}$, Gregory \\ R Steinberg ${ }^{3}$, Bruce E Kemp ${ }^{3,4}$, Robert J Southgate ${ }^{1}$, Graeme I Lancaster ${ }^{1}$, Clinton R Bruce ${ }^{1}$, Matthew \\ J Watt ${ }^{5}$ and Mark A Febbraio ${ }^{1}$ \\ ${ }^{1}$ Diabetes and Metabolism Division, Cellular and Molecular Metabolism Laboratory, Baker Heart Research Institute, PO Box 6492, St Kilda Road Central, \\ Melbourne, Victoria 8008, Australia \\ ${ }^{2}$ School of Medical Sciences, RMIT University, Bundoora, Victoria 3083, Australia \\ ${ }^{3}$ St Vincent's Institute and Department of Medicine, The University of Melbourne, Fitzroy, Victoria 3065, Australia \\ ${ }^{4}$ CSIRO Molecular and Health Technologies, 343 Royal Parade, Parkville, Victoria 3052, Australia \\ ${ }^{5}$ Department of Physiology, Monash University, Clayton, Victoria 3800, Australia \\ (Correspondence should be addressed to M A Febbraio; Email: mark.febbraio@baker.edu.au) \\ (J L Mesa is currently at the Medical Research Centre, Epidemiology Unit, Cambridge, UK) \\ *(A G Holmes and J L Mesa contributed equally to this work)
}

\begin{abstract}
Chronic elevations in interleukin (IL)-6 have been associated with insulin resistance, but acute IL-6 administration can enhance insulin sensitivity. Our aim was to exogenously administer IL-6 to rats to elicit either chronic or repeated acute elevations in systemic IL-6. We hypothesized that a continuous elevation of IL-6 would inhibit glucose tolerance and insulin sensitivity while acute intermittent elevations would improve it. Male Wistar rats were treated for $14 \mathrm{~d}$ with recombinant human IL-6 $(2 \cdot 4 \mu \mathrm{g} /$ day $)$ or saline administered either by miniosmotic pump (continuous IL-6) or via twicedaily injection (intermittent IL-6). Glucose and insulin tolerance tests were performed following 14-d treatment and $24 \mathrm{~h}$ later rats were administered a bolus of insulin $(150 \mathrm{mU} / \mathrm{g})$ or saline intraperitoneally. Approximately, $10 \mathrm{~min}$ after insulin injection soleus, gastrocnemius and liver were excised and
\end{abstract}

rapidly frozen in liquid nitrogen for subsequent metabolic measures. Irrespective of the mode of delivery, IL-6 treatment increased basal insulin sensitivity, as measured by the homeostatic model assessment of insulin resistance, and enhanced glucose clearance during an i.p. glucose tolerance test. IL-6 increased circulating fatty acids, but did not increase triglyceride accumulation in either skeletal muscle or liver, while it increased the protein expression of both PPAR $\alpha$ and UCP2 in skeletal muscle, suggesting that IL-6 can enhance fat oxidation via mitochondrial uncoupling. These data demonstrate that, irrespective of the mode of delivery, IL-6 administration over 2 weeks enhances glucose tolerance. Our results do not support the notion that prolonged chronically elevated IL-6 impairs insulin action in vivo.

Journal of Endocrinology (2008) 198, 367-374

\section{Introduction}

In the past decade, it has become apparent that obesity is linked to a state of chronic inflammation that occurs in tissue such as the liver, adipose and skeletal muscle (Hotamisligil 2006). Obesity results in the secretion of inflammatory cytokines such as resistin, tumour necrosis factor- $\alpha$ and interleukin-6 (IL-6) form macrophages and/or adipocytes, which can then act via transmembrane receptors in metabolically active tissue to activate serine/threonine kinases known to disrupt insulin signal transduction (Wellen \& Hotamisligil 2005). Given this pro-inflammatory response, and the observation that systemic IL-6 concentrations are elevated in obesity and patients with type 2 diabetes (Bastard et al. 2000, Vozarova et al. 2001,
Carey et al. 2004), it is generally thought that elevations in the plasma and/or tissue concentrations of IL-6 have a negative effect on metabolism (Lazar 2005). However, the discovery that IL-6 can be produced (Hiscock et al. 2004) and released (Steensberg et al. 2001) from skeletal muscle during exercise, led to renewed interest in the role of IL-6 in the aetiology of insulin resistance because insulin action is enhanced in the immediate post-exercise period (Wojtaszewski et al. 2000). Presently, the role of IL-6 in insulin resistance is both unclear and the subject of intense debate (Carey \& Febbraio 2004, Kristiansen \& Mandrup-Poulsen 2005, Ruderman et al. 2006, Mooney 2007, Pedersen \& Febbraio 2007). On the one hand, subjecting lean mice to chronically elevated IL-6 for 5 days causes hepatic insulin resistance (Klover et al. 2003), while treating mice 
that harbour genetic mutations resulting in hepatic insulin resistance, with IL-6 neutralizing antibodies improves such a phenotype (Cai et al. 2005, Klover et al. 2005). On the other hand, several recent studies have demonstrated that IL-6 can enhance both glucose uptake (Al-Khalili et al. 2006, Carey et al. 2006, Glund et al. 2007) and fat oxidation (Bruce \& Dyck 2004, Petersen et al. 2005, Al-Khalili et al. 2006), principally via the activation of AMP-activated protein kinase (AMPK) (Carey et al. 2006), while IL-6 knockout mice become obese and glucose intolerant (Wallenius et al. 2002).

While it is difficult to reconcile the lack of consensus regarding the role of IL-6 on insulin action, in general, it appears that in circumstances where IL- 6 is chronically elevated, it has a negative effect on insulin action while acute IL-6 treatment tends to improve insulin action. This may be due to the fact that chronically elevated IL-6 results in elevated expression of suppressor of cytokine signalling-3 (SOCS3) (Klover et al. 2003), which is generally thought to induce insulin resistance (Ueki et al. 2004), while acute doses of IL-6 may not result in a sustained increase in SOCS3 necessary to disrupt insulin signalling. Accordingly, in the present study we tested the hypothesis that chronic elevation of IL-6 may induce insulin resistance, but acute IL-6 elevations may result in an insulinsensitizing effect due to the activation of AMPK. To test this hypothesis, we treated male Wistar rats for $14 \mathrm{~d}$ with recombinant human (rh) IL-6 $(2 \cdot 4 \mu \mathrm{g}$ /day) or saline administered either by miniosmotic pump (continuous IL-6) or via twice-daily injection (intermittent IL-6). Irrespective of the mode of delivery, herein we show that 14-d treatment with IL-6 improves insulin sensitivity and glucose tolerance.

\section{Materials and Methods}

\section{Animals}

Male Wistar rats weighing $\sim 220 \mathrm{~g}$ were purchased from Monash Animal Facility (Clayton, Victoria, Australia). The animals were housed in the RMIT University animal facility in a controlled environment with a ratio of $12 \mathrm{~h}$ light: $12 \mathrm{~h}$ darkness cycle and were fed rat food and water ad libitum. All experimental procedures were approved by the Animal Ethics Committee of RMIT University. Animals were randomly assigned to receive IL-6 or saline vehicle in a continuous or intermittent manner, with 12 animals in each group.

\section{IL-6 treatment}

For experiments examining continuous IL-6 exposure, osmotic pumps (Alzet 2ML2; Durect, Cupertino, CA, USA) with a 14-d pumping capacity and an infusion rate of $4.5 \mu \mathrm{l} / \mathrm{h}$ were used. Pumps were filled to capacity with $22.5 \mu \mathrm{g} / \mathrm{ml}$ rhIL-6 diluted in carrier $(0.9 \% \mathrm{NaCl}$ and $0.1 \% \mathrm{BSA})$ resulting in a daily delivery of $2400 \mathrm{ng}$ rhIL-6. Following the induction of general anaesthesia with sodium pentobarbitone $(6 \mathrm{mg} / 100 \mathrm{~g}$ bw; Sigma), pumps were implanted into the intrascapular subcutaneous space. Incisions were closed with interrupted absorbable sutures. Control animals received pumps containing the carrier alone. Intermittent IL- 6 treatment was achieved by injecting rats with rhIL-6 into the intraperitoneal space twice daily. At 0700 and 1900 h, 1200 ng rhIL-6 diluted in carrier $(0 \cdot 9 \% \mathrm{NaCl}$ and $0 \cdot 1 \% \mathrm{BSA})$ was injected daily for $14 \mathrm{~d}$. Control animals received saline injections of the same volume at the same time. Pumps were removed $24 \mathrm{~h}$ prior to metabolic testing. Due to the large quantities of recombinant IL-6 necessary to perform these experiments, it was not possible to use recombinant mouse IL-6 and we were, therefore, compelled to use rhIL-6. However, rhIL-6 has been shown to be as effective in rodent as in human cells (Cayphas et al. 1987) and rhIL-6 has been used in previous rodent in vivo experiments with efficacy (Klover et al. 2003).

\section{Insulin and glucose tolerance tests}

Rats were fasted for $12 \mathrm{~h}$ overnight and tested at 0700. Basal blood samples $(t=0)$ were taken from the tail of restrained rats for blood glucose and plasma insulin measures. For glucose tolerance tests, $1 \mathrm{~g} / \mathrm{kg}$ bw glucose was injected i.p. Blood samples for the measurement of glucose and plasma insulin levels were obtained from the tail at 15, 30,60 and $120 \mathrm{~min}$. For insulin tolerance tests, $0.75 \mathrm{U} / \mathrm{kg}$ bw insulin (rh insulin; Actrapid) was injected i.p. Blood samples for glucose measurement were obtained from the tail at 15, 30, 60 and $120 \mathrm{~min}$.

\section{Tissue collection and analyses}

Rats were anaesthetized (6 mg/100 g bw sodium pentobarbitone; Sigma), and injected with insulin $(150 \mathrm{U} / \mathrm{kg})$ or saline vehicle i.p. After $\sim 10$ min soleus, gastrocnemius and liver were excised and rapidly frozen in liquid nitrogen for storage at $-80{ }^{\circ} \mathrm{C}$. Muscle and liver triacylglycerol (TAG) content were analyzed using the Folch extraction method as previously described (Bruce et al. 2003). Maximal activities of the enzymes citrate synthase (CS) and $\beta$-hydroxyAcyl-CoA dehydrogenase $(\beta-H A D)$ in liver and skeletal muscle were assayed spectrophotometrically (Watt et al. 2006a). AMPK activity in muscle and liver were analysed using the AMPK SAMS peptide assay previously described (Watt et al. 2006b). Proteins were analysed by western blot as previously described (Carey et al. 2006, Watt et al. 2006a). All antibodies except for anti-UCP3, anti-UCP2 (Alpha Diagnostics, San Antonio, TX, USA), anti-

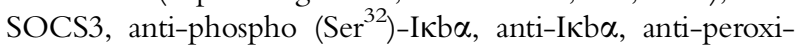
some proliferator-activated receptor (PPAR) $\gamma$ coactivator-1 $\alpha$ (PGC-1 $\alpha$ ) (Santa Cruz Biotechnology, Santa Cruz, CA, USA), anti-PPAR $\delta$ and anti-PPAR $\alpha$ (Research Diagnostics, Concord, MA, USA) were obtained from Cell Signaling (Beverly, MA, USA).

\section{Plasma analysis}

Plasma IL-6 was measured by commercially available enzymelinked immunosorbent assay (Rat ELISA Cat No BMS625, Bender MedSystems Inc., Burlingame, CA, USA). Plasma 
glucose was measured using an automated glucose-oxidase method (Yellow Springs Instruments, Yellow Springs, OH, USA). Insulin was measured by ELISA (\#EZRMI-13K, Linco Research, St. Charles, MO, USA). The homeostatic model assessment of insulin resistance (HOMA-IR) was then calculated from these results $($ HOMA-IR $=$ plasma insulin $(\mu \mathrm{U}) \times$ glucose $(\mathrm{mmol} / \mathrm{l}) / 22 \cdot 5)$. Free fatty acid content (FFA) was measured spectrophotometrically by an enzymatic colorimetric assay (Wako NEFA C kit; Wako Chemicals, Richmond, VA, USA).

\section{Statistical analyses}

Data were analyzed using a one-way or two-way ANOVA with repeated measures (SPSS, Chicago, IL, USA), with significance accepted with a $P$ value of $<0 \cdot 05$. If analyses revealed a significant interaction, a Newman-Keuls post hoc test was used to locate specific differences. Data are presented as means \pm S.E.M.

\section{Results}

No differences in any measures were observed between intermittent saline- and continuous saline-treated groups. Hence, data from these two groups were pooled and are herein referred to as control. No differences were observed in body weight, food intake or adipose tissue mass when comparing the three groups over the two-week period. IL-6 concentration in control was $23 \cdot 8 \pm 8 \cdot 8$ vs $134 \pm 69 \mathrm{pg} / \mathrm{ml}$ in continuous IL-6 treated rats. Importantly, this approximate sixfold elevation of IL-6 attained with continuous IL-6 was similar to levels reached in obesity, as previously reported (Klover et al. 2003). During intermittent IL-6 treatment, IL-6 steadily increased reaching a peak of $622 \pm 280 \mathrm{pg} / \mathrm{ml}, 3 \mathrm{~h}$ following injection after which time it fell back returning to baseline $5 \mathrm{~h}$ after injection. This represented an approximate 30-fold increase in IL-6 and it has previously been shown that during strenuous exercise IL-6 can increase over 100-fold in the plasma (Starkie et al. 2001).

\section{IL-6 treatment decreases HOMA-IR and increases glucose tolerance}

Fasting plasma glucose levels averaged $5 \cdot 0 \pm 0 \cdot 2,4 \cdot 4 \pm 0 \cdot 4$ and $3.9 \pm 0 \cdot 1 \mathrm{mmol} / 1$ not significant (NS) for control, intermittent IL-6 and continuous IL-6 respectively. Fasting plasma insulin levels averaged $44 \cdot 0 \pm 7 \cdot 7,18 \cdot 5 \pm 4 \cdot 3$ and $25 \cdot 9 \pm 8 \cdot 7 \mathrm{pmol} / 1$ (NS) for control, intermittent IL-6 and continuous IL-6 respectively. Based on these values, we calculated the HOMAIR. Irrespective of the mode of delivery, IL-6 treatment decreased $(P<0 \cdot 05)$ HOMA-IR (Fig. 1A). Next, to assess whether IL-6 affected glucose or insulin tolerance, we
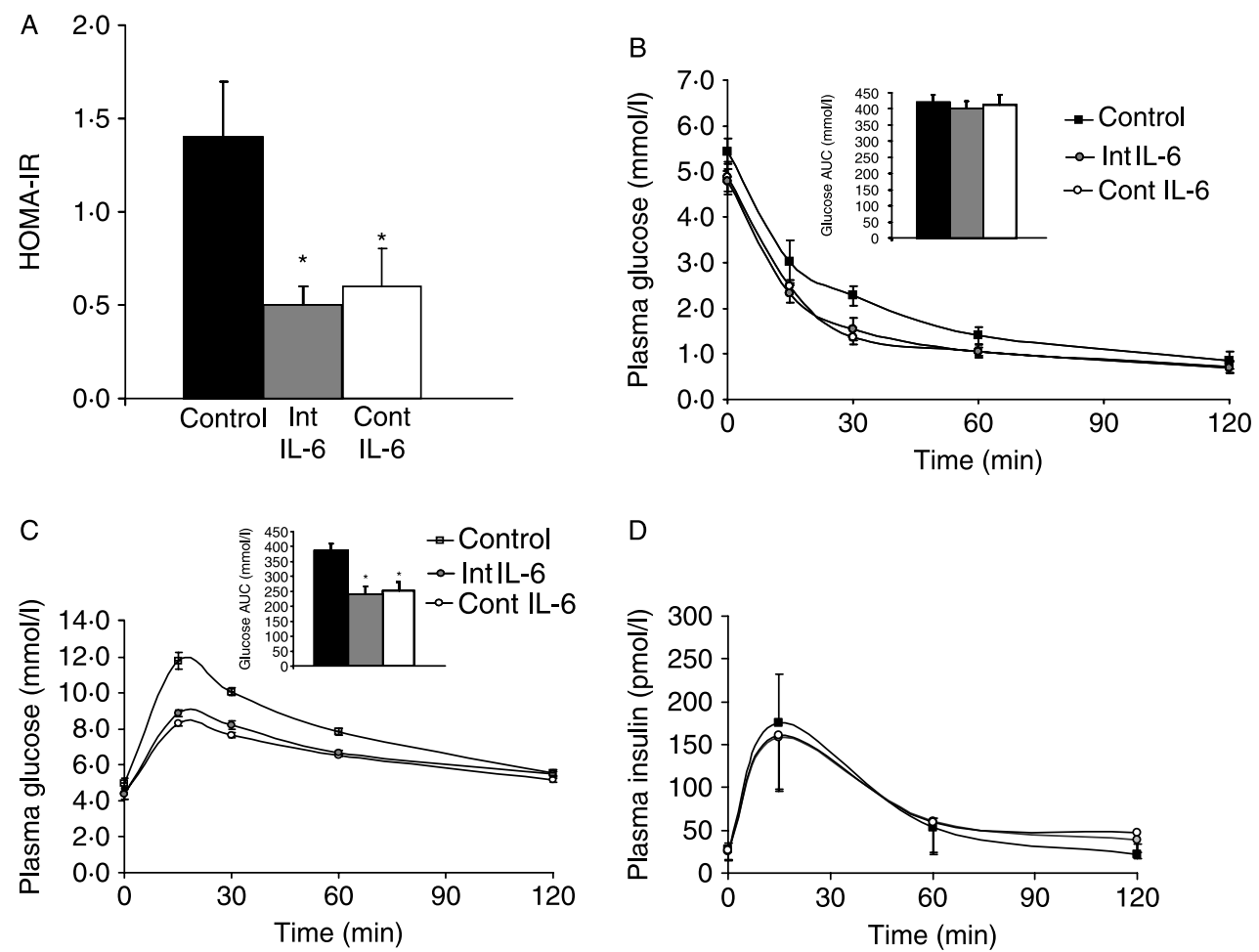

Figure 1 (A) Homeostatic model assessment of insulin resistance (HOMA-IR), (B) plasma glucose levels during an i.p. insulin, (C) glucose tolerance test and (D) plasma insulin levels during an i.p. insulin tolerance test in rats treated for $14 \mathrm{~d}$ with vehicle control (control) or $2 \cdot 4 \mu \mathrm{g} /$ day interleukin-6 administered either by miniosmotic pump (continuous IL-6) or via twice-daily injection (intermittent IL-6). Data are expressed as mean \pm S.E.M., $n=8-10$ animals per group, asterisk indicates difference $(P<0 \cdot 05)$ from control. 
performed intraperitoneal insulin tolerance test (IPITT) and IPGTT. Somewhat surprisingly we observed no effect of IL-6 on glucose concentration during the IPITT (Fig. 1B). However, consistent with our HOMA-IR measures, both intermediate and continuous IL-6 treatments markedly improved glucose tolerance (Fig. 1C). This was unlikely due to an effect on insulin secretion as no differences were observed in insulin concentration at anytime point during the IPGTT (Fig. 1D). Given the results with respect to HOMA-IR and glucose tolerance, we next assessed insulin signalling by measuring the tyrosine phosphorylation of insulin receptor substrate-1 (IRS-1), the association of IRS-1 with the p85 subunit of phosphatidylinositol-3 kinase (PI3)-kinase and phosphorylation of Akt $\left(\mathrm{Ser}^{473}\right)$. Although, as expected, insulin markedly increased these measures, IL- 6 treatment had no effect on any aspect of insulin signalling in either tissue (Fig. 2A-C).

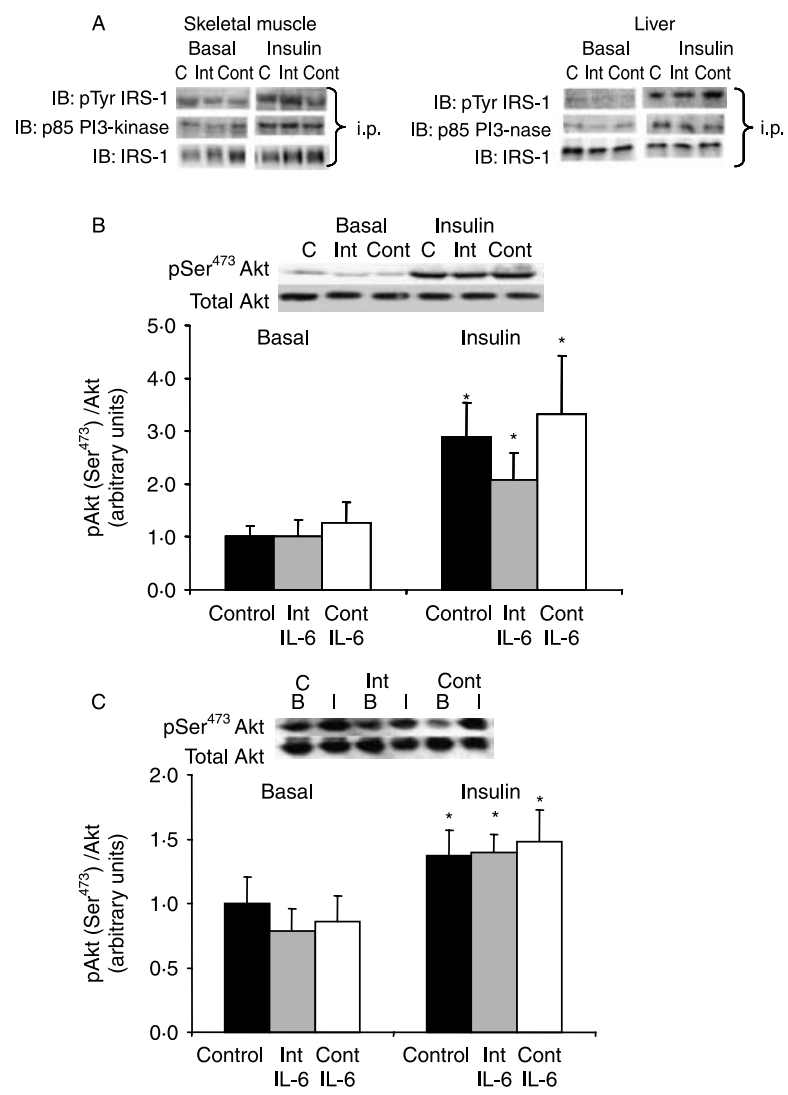

Figure 2 Representative immunoblots of tyrosine phosphorylation of IRS-1, the association of the p85 subunit of PI3-kinase with IRS-1 and total IRS-1 in (A) skeletal muscle and liver, (B) quantification of phosphorylated $\left(\mathrm{Ser}^{473}\right) /$ total Akt in skeletal muscle and (C) liver of rats treated for $14 \mathrm{~d}$ with vehicle control (control) or $2 \cdot 4 \mu \mathrm{g} /$ day interleukin- 6 administered either by miniosmotic pump (continuous IL-6) or via twice-daily injection (intermittent IL-6). Rats injected with saline (Basal) or $150 \mathrm{mU} / \mathrm{g}$ insulin (Insulin) 10 min prior to excision of tissues under anaesthesia. Data are expressed as mean \pm S.E.M., $n=8-10$ animals per group, asterisk indicates difference $(P<0 \cdot 05)$ Insulin versus basal.
IL-6 treatment increases circulating FFA, but does not affect muscle or liver TAG content

Acute IL-6 treatment has been shown to increase both adipose tissue lipolysis and skeletal muscle fat oxidation in vitro (Peterson et al. 2005, Carey et al. 2006) and in humans (van Hall et al. 2003, Peterson et al. 2005) and rodents (Bruce \& Dyck 2004) in vivo. Although in the present study, we did not measure fat oxidation, we measured circulating FFA and skeletal muscle and liver TAG content. Consistent with the notion that IL-6 increases lipolysis, both intermittent and continuous IL-6 treatments markedly
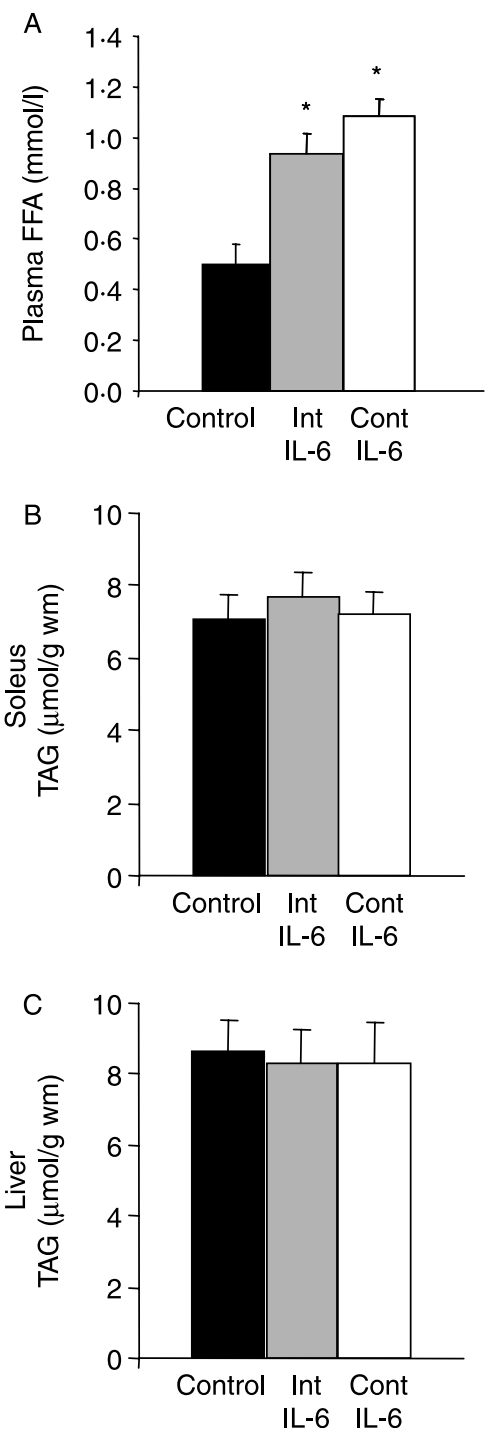

Figure 3 (A) Plasma free fatty acids and (B) triacylglycerol content in skeletal muscle and (C) liver from rats treated for $14 \mathrm{~d}$ with vehicle control (control) or $2 \cdot 4 \mu \mathrm{g}$ /day interleukin- 6 administered either by miniosmotic pump (continuous IL-6) or via twice-daily injection (intermittent IL-6). Data are expressed as mean \pm S.E.M., $n=8-10$ animals per group, asterisk indicates difference $(P<0 \cdot 05)$ from control. 
increased fasting plasma FFA concentration (Fig. 3A). Despite this elevation, we observed no differences in TAG accumulation in either skeletal muscle (Fig. 3B) or liver (Fig. 3C), suggesting that IL-6 may have increased fat oxidation.

\section{IL-6 increases PPAR $\alpha$ and UCP2 expression in skeletal muscle}

Carey et al. (2006) and others (Kelly et al. 2004, Al-Khalili et al. 2006, Glund et al. 2007) have reported that acute IL-6 treatment increases the activation of AMPK in skeletal muscle. Moreover, IL-6-induced increases in fat oxidation appear to be AMPK dependent (Carey et al. 2006). Accordingly, we next examined the effect of intermittent and continuous IL-6 treatment on AMPK activity in skeletal muscle and liver. By contrast, with the acute effects of IL-6 on AMPK, we saw no differences when comparing IL-6 treatment with control in either skeletal muscle (Fig. 4A) or liver (Fig. 4B). These data suggest that the effects of IL-6 on increasing AMPK activity are acute and do not persist $24 \mathrm{~h}$ after IL-6 stimulation has ceased. Consistent with this notion, we saw no differences when comparing the three groups for the phosphorylation of STAT3 $\left(\mathrm{Tyr}^{705}\right.$ ) in skeletal muscle and we have previously shown a relationship between STAT3 phosphorylation and AMPK activation when treating animals with gp130 receptor cytokines (Carey et al. 2006, Watt et al. 2006a). To next test whether IL-6 may have up-regulated key
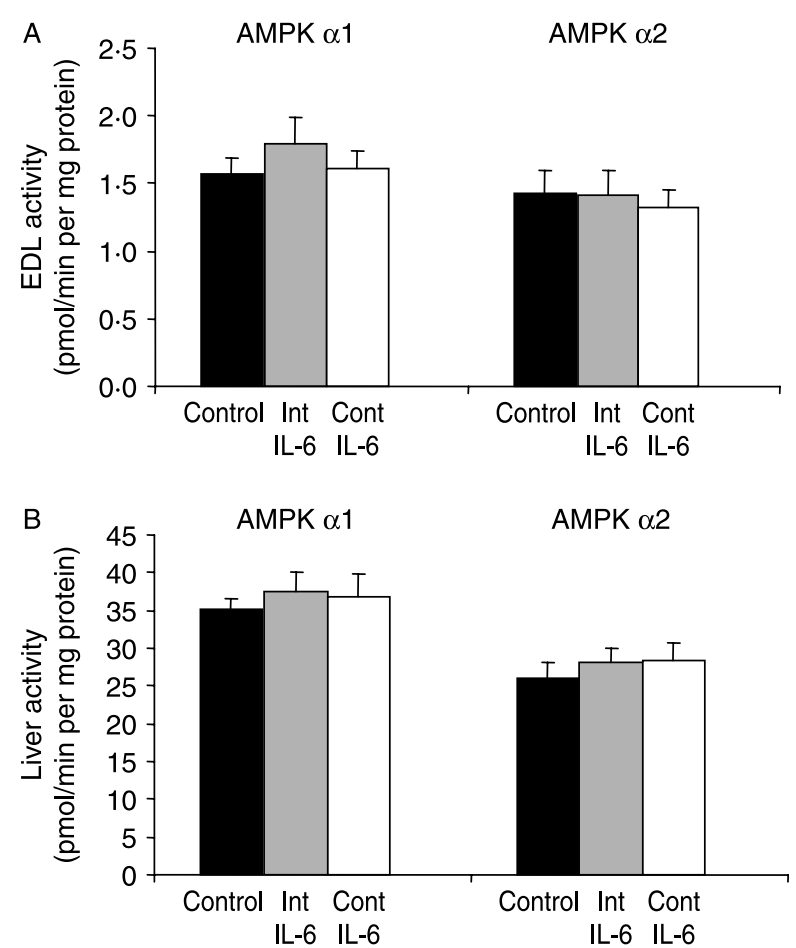

Figure 4 AMPK $\alpha 1$ and $\alpha 2$ activity in (A) EDL muscle and (B) liver from rats treated for $14 \mathrm{~d}$ with vehicle control (control) or $2.4 \mu \mathrm{g}$ /day interleukin- 6 administered either by miniosmotic pump (continuous IL-6) or via twice-daily injection (intermittent IL-6). Data are expressed as mean \pm s.E.M., $n=8-10$ animals per group. pathways implicated in fat metabolism and enhanced insulin sensitivity, we measured a number of proteins. We observed no difference in GLUT4 protein expression in skeletal muscle when comparing the three groups (data not shown). The expression of PGC-1 $\alpha$ (Handschin \& Speigelman 2006) and PPARs (Evans et al. 2004) have been directly implicated in fat metabolism and insulin sensitivity. Accordingly, we measured PGC- $1 \alpha$ and PPAR isoforms in skeletal muscle after IL-6 treatment. Irrespective of mode of delivery, IL-6 did not affect the expression of PGC-1 $\alpha$ or PPAR $\delta$ (Fig. 5A). However, irrespective of mode of delivery, IL-6 treatment increased the expression of PPAR $\alpha$ protein expression in skeletal muscle (Fig. 5A). The uncoupling proteins UCP2 and UCP3 have also been implicated in increasing substrate oxidation (Schrauwen \& Hesselink 2002), and UCP2 expression is reduced in skeletal muscle from obese patients (Nordfors et al. 1998). Accordingly, we measured the protein expression of both UCP2 and UCP3 in the skeletal muscle of our treated rats. While UCP3 was not affected by IL-6 treatment (Fig. 5B), UCP2 expression was elevated in both phasic and chronic IL-6 treatment (Fig. 5B).

Since we were unable to measure lipid oxidation and since PPAR and UCPs have been linked to enhanced oxidative
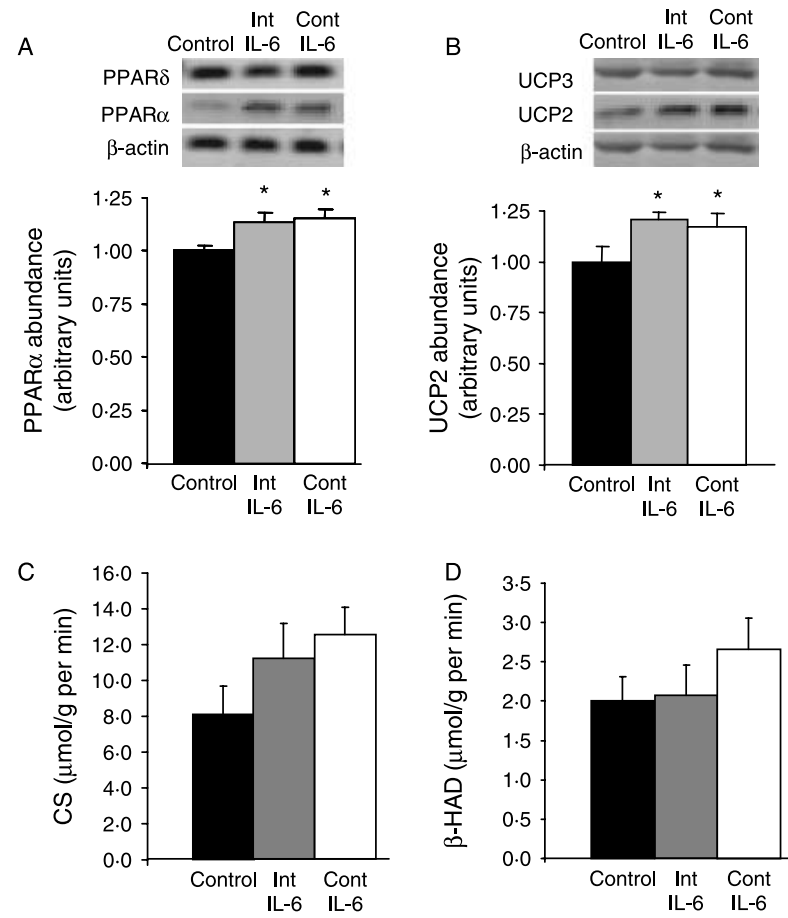

Figure 5 Representative immunoblot of (A) PPAR $\delta$ and (B) UCP3, (A) quantification of PPAR $\alpha / \beta$-Actin, (B) UCP2/ $\beta$-Actin, (C) maximal activity of citrate synthase (CS) and (D) $\beta$-hydroxyAcyl-CoA dehydrogenase $(\beta-H A D)$ in skeletal muscle from rats treated for $14 \mathrm{~d}$ with vehicle control (control) or $2 \cdot 4 \mu \mathrm{g} /$ day interleukin- 6 administered either by miniosmotic pump (continuous IL-6) or via twice daily injection (intermittent IL-6). Data are expressed as mean \pm S.E.M., $n=8-10$ animals per group, asterisk indicates difference $(P<0 \cdot 05)$ from control. 
metabolism, we next measured the maximal activity of the enzymes $\mathrm{CS}$ and $\beta-\mathrm{HAD}$ that are the important markers of mitochondrial capacity. No differences were observed in liver CS and $\beta$-HAD maximal activities (data not shown). Although there was a tendency in skeletal muscle for both CS and $\beta$-HAD to be increased with continuous IL-6 treatment when compared with control when analyzed by ANOVA, results were not significant. Of, note, however, compared by $t$-test, $\beta$-HAD maximal activity was higher $(P<0 \cdot 05)$ when comparing continuous IL-6 with control.

IL-6 treatment does not result in SOCS3 or activation of the NFKB signalling cascade in skeletal muscle or liver.

As discussed, previous studies have shown that IL-6 impairs hepatic insulin signalling (Klover et al. 2005) which has been linked to elevated liver SOCS3 expression (Klover et al. 2003). In addition, recent evidence has suggested that IL-6 may mediate NFKB-induced insulin resistance (Cai et al. 2004). Accordingly, in the present study, we measured SOCS3 mRNA and protein expression and I $\mathrm{BB}$ phosphorylation (Ser ${ }^{32 / 36}$ ) and expression in both liver and skeletal muscle. SOCS3 mRNA abundance was elevated in skeletal muscle (Fig. 6A), but not in liver (Fig. 6B) compared with control. However, we observed no differences when comparing groups for SOCS3 protein expression and/or phosphorlyated or total $\mathrm{I} \kappa \mathrm{B} \alpha$ in either skeletal muscle or liver (Fig. 6C).

\section{Discussion}

The results from this study demonstrate that irrespective of mode of delivery, IL-6 administration for $14 \mathrm{~d}$ increases

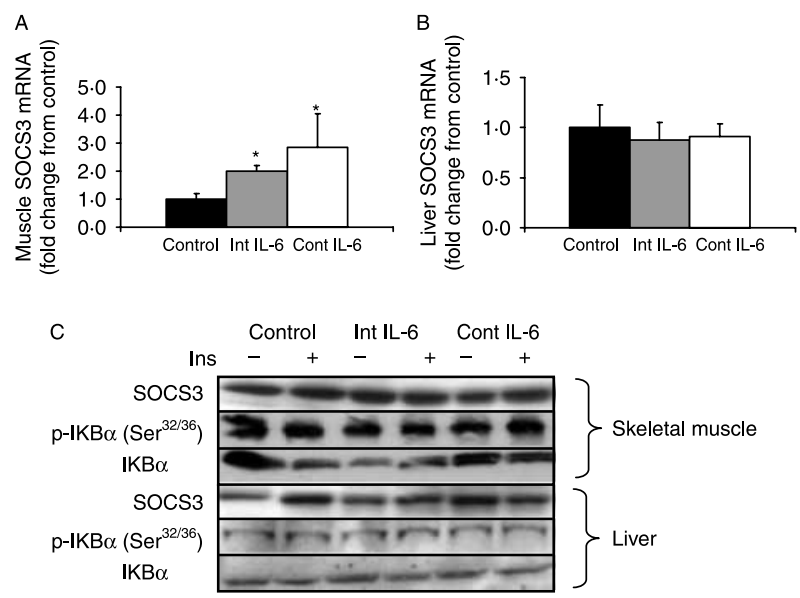

Figure 6 SOCS3 mRNA in (A) skeletal muscle, (B) liver and (C) representative immunoblot of SOCS3 protein and phosphorylated $\left(\mathrm{Ser}^{32 / 36}\right) /$ total IKB $\alpha$ protein in skeletal muscle and liver from rats treated for $14 \mathrm{~d}$ with vehicle control (control) or $2 \cdot 4 \mu \mathrm{g} /$ day interleukin- 6 administered either by miniosmotic pump (continuous IL-6) or via twice-daily injection (intermittent IL-6). Data are expressed as mean \pm S.E.M., $n=8-10$ animals per group, asterisk indicates difference $(P<0 \cdot 05)$ from control. glucose tolerance in healthy rats. Moreover, such treatment increases the protein expression of $\operatorname{PPAR} \alpha$ and UCP2 in skeletal muscle, two proteins that have been implicated in the upregulation of fat oxidation.

Our data are in contrast to those reported by Mooney et al. in previous studies in mice (Klover et al. 2003). We have no direct explanation why our data differs with this previous report, however, there are some important methodological differences when comparing our study with this previous report. Firstly, our study was performed in rats and we have noted when comparing rats, mice and humans during euglycemic hyperinsulinemic clamps (EHC) that while humans and rats exhibit $\sim 100 \%$ suppression of hepatic glucose production during EHC, this is not so with mice, suggesting that mice display liver insulin resistance relative to the other species (Febbraio unpublished observations). Therefore, while both studies were performed in rodents, the species difference may be important. It is also important to note that in our study, we chose to remove the miniosmotic pumps or deliver the last IL-6 injection $24 \mathrm{~h}$ prior to metabolic testing whereas in the previous paper (Klover et al. 2003), it appeared that the IL-6 treatment persisted throughout. We chose this strategy in an attempt to test whether the chronic effects of IL-6 persist in the absence of acute IL-6 stimulation and this may have been a crucial methodological difference when comparing the two studies.

In the present study, we demonstrated that IL-6 treatment lowered basal glucose and insulin levels (as measured by HOMA-IR) and markedly improved glucose tolerance. Consistent with these results, in a previous publication from our group, we also observed a marked drop in both circulating insulin and HOMA-IR during IL-6 infusion into humans (Petersen et al. 2005). However, in the present study, we did not detect differences in insulin tolerance as measured during the IPITT or improved insulin signalling as measured by tyrosine phosphorylation of IRS-1, the association of IRS-1 with the p85 subunit of PI3-kinase or the phosphorylation of Akt $\left(\mathrm{Ser}^{473}\right)$ in liver or muscle. It is unlikely that the improved glucose tolerance was due to an effect of IL-6 on insulin secretion during the IPGTT, since we measured near identical plasma insulin levels during this test (Fig. 1D). We have no explanation why we would observe differences in HOMA-IR and GTT, but not ITT or insulin signal transduction although it must be noted that in our ITT, the animals were relatively insulin tolerant as the glucose levels fell precipitously with the nadir being $\sim 5$ fold lower than basal (Fig. 1B). It is possible that this dose of insulin $(0 \cdot 75 \mathrm{U} / \mathrm{kg} \mathrm{bw})$ was too severe and induced counter-regulatory measures which masked any difference observed in insulin tolerance, but this is speculative. In previous studies, we have measured an increase in insulinstimulated glucose uptake and phosphorylation of Akt in muscle cells exposed to acute (120 min) IL-6 (Carey et al. 2006). It was not possible to chronically treat muscle cells for the period of time that we exposed the rats to IL-6, however, in attempting to elucidate the mechanism by which chronic IL-6 treatment lead to enhanced glucose tolerance, we treated 
L6 myotubes with $100 \mathrm{ng} / \mathrm{ml} \mathrm{IL-6} \mathrm{for} \mathrm{1,} \mathrm{12,} 24$ and $48 \mathrm{~h}$. Of note, the enhanced insulin action we previously observed when cells were exposed to IL-6 for $120 \mathrm{~min}$ (Carey et al. 2006) was also seen in these recent experiments at 1 and $12 \mathrm{~h}$, but were abolished at 24 and $48 \mathrm{~h}$ (data not shown). Together, these data coupled with the ITT (Fig. 1) and insulin signalling data (Fig. 2), would argue against chronic IL-6 enhancing insulin action in muscle. Hence, the mechanism accounting for the enhanced glucose tolerance that was observed in every animal irrespective of mode of delivery requires further investigation.

IL-6 rapidly induces the transient expression of SOCS3 (Starr et al. 1997). In the present study, we observed an increase in SOCS3 mRNA in skeletal muscle (Fig. 6A) but not in liver (Fig. 6B), but, importantly no elevation in SOCS3 protein expression in either liver or skeletal muscle $24 \mathrm{~h}$ following removal of IL-6 stimulation (Fig. 6C), indicating that the IL-6 induced elevation in SOCS3 is transient. While we have previously reported that treating skeletal muscle myotubes and adipocytes with IL-6 results in elevated SOCS3 expression within $60 \mathrm{~min}$ of the treatment (Carey et al. 2006), without altering insulin sensitivity, others have shown that the IL-6 mediated insulin resistance in hepatocytes was associated with increased SOCS3 expression (Senn et al. 2003). The notion that SOCS3 induces insulin resistance (Ueki et al. 2004) has recently been challenged by two in vivo studies. Firstly, while liver specific knockout mice display enhanced liver insulin signalling, they become obese and insulin resistant, suggesting that SOCS3 expression in liver functions to suppress inflammation and systemic insulin resistance (Torisu et al. 2007). Secondly, Inoue et al. (2006) have reported that IL-6-STAT3 signalling in the liver, which results in elevated SOCS3 expression, contributes to insulin action in the brain, leading to the suppression of hepatic glucose production. Hence, the notion that IL-6 may mediate hepatic insulin resistance by increasing hepatic SOCS3 expression is unclear and requires further work.

IL-6 increased circulating FFA without altering liver and skeletal muscle TAG accumulation. These data coupled with the observed increases in PPAR $\alpha$ and UCP2 expression in skeletal muscle suggests that IL-6 increased fatty acid oxidation. In addition, the tendency for chronic IL-6 administration to increase the maximal activity of CS and $\beta-\mathrm{HAD}$ is consistent with an effect of IL-6 on increasing mitochondrial capacity and hence the ability to oxidize substrate. These data are consistent with previous reports that IL-6 increases lipolysis and lipid oxidation in vitro, in vivo and in skeletal muscle ex vivo (van Hall et al. 2003, Bruce \& Dyck 2004, Petersen et al. 2005).

In summary, we have demonstrated that irrespective of the mode of delivery IL-6 administered to rats for $14 \mathrm{~d}$ increases basal insulin sensitivity, as measured by the HOMA-IR and enhances glucose clearance during an IPGTT. Moreover, while IL-6 increased circulating fatty acids, it did not increase lipid accumulation in either skeletal muscle or liver while it increased the protein expression of both PPAR $\alpha$ and UCP2 in skeletal muscle. These data demonstrate that, irrespective of mode of delivery, IL-6 administration over 2 weeks enhances glucose tolerance. Our results do not support the notion that prolonged chronically elevated IL-6 impairs insulin action in vivo.

\section{Declaration of Interest}

The authors declare that there is no conflict of interest that would prejudice the impartiality of this scientific work.

\section{Funding}

This work was supported by a grant from the National Health and Medical Research Council of Australia (NHMRC 342115). A G H was and B A N is supported by an Australian Post graduate award, A L C and C R B are supported by Peter Doherty Post Doctoral Fellowships (NHMRC). B E K is an Australian Research Council (ARC) Federation Fellow, R J S is an ARC APD Fellow, $M \mathrm{~J} \mathrm{~W}$ is an RD Wright Fellow (NHMRC), and M A F is a Principal Research Fellow (NHMRC). J L M was a visiting student from The University of Grenada, Spain.

\section{References}

Al-Khalili L, Bouzakri K, Glund S, Lönnqvist F, Koistinen HA \& Krook A 2006 Signaling specificity of interleukin-6 action on glucose and lipid metabolism in skeletal muscle. Molecular Endocrinology 20 3364-3375.

Bastard JP, Jardel C, Bruckert E, Blondy P, Capeau J, Laville M, Vidal H \& Hainque B 2000 Elevated levels of interleukin 6 are reduced in serum and subcutaneous adipose tissue of obese women after weight loss. Journal of Clinical Endocrinology and Metabolism 85 3338-3342.

Bruce CR \& Dyck DJ 2004 Cytokine regulation of skeletal muscle fatty acid metabolism: effect of interleukin-6 and tumor necrosis factor-alpha. American Journal of Physiology. Endocrinology and Metabolism 287 E616-E621.

Bruce CR, Carey AL, Hawley JA \& Febbraio MA 2003 Intramuscular HSP72 and $\mathrm{HO}-1$ mRNA are reduced in patients with type 2 diabetes: evidence that insulin resistance is associated with a disturbed anti-oxidant defense mechanism. Diabetes 52 2338-2345.

Cai D, Yuan M, Frantz DF, Melendez PA, Hansen L, Lee J \& Shoelson SE 2005 Local and systemic insulin resistance resulting from hepatic activation of IKK-beta and NF-kB. Nature Medicine 11 183-190.

Carey AL \& Febbraio MA 2004 Interleukin-6 and insulin sensitivity: friend or foe? Diabetologia 47 1135-1142.

Carey AL, Bruce CR, Sacchetti M, Anderson MJ, Olson DB, Saltin B, Hawley JA \& Febbraio MA 2004 IL-6 and TNF $\alpha$ are not elevated in patients with type two diabetes: evidence that plasma IL-6 is related to fat mass and not insulin responsiveness. Diabetologia 47 1029-1037.

Carey AL, Steinberg GR, Macaulay SL, Thomas WJ, Holmes AG, Ramm G, Prelovsek O, Hohnen-Behrens C, Watt MJ, James DE et al. 2006 Interleukin-6 increases insulin-stimulated glucose disposal in humans and glucose uptake and fatty acid oxidation in vitro via AMP-activated protein kinase. Diabetes 55 2688-2697.

Cayphas S, Van Damme J, Vink A, Simpson RJ, Billiau A \& Van Snick J 1987 Identification of an interleukin HP1-like plasmacytoma growth factor produced by L cells in response to viral infection. Journal of Immunology 139 2965-2969.

Evans RM, Barish GD \& Wang YX 2004 PPARs and the complex journey to obesity. Nature Medicine 104 355-361.

Glund S, Deshmukh A, Long YC, Moller T, Koistinen HA, Caidahl K, Zierath JR \& Krook A 2007 Interleukin-6 directly increases glucose metabolism in resting human skeletal muscle. Diabetes 56 1630-1637. van Hall G, Steensberg A, Sacchetti M, Fischer C, Keller C, Schjerling P, Hiscock N, Moller K, Saltin B, Febbraio MA et al. 2003 Interleukin-6 stimulates lipolysis and fat oxidation in humans. Journal of Clinical Endocrinology and Metabolism 88 3005-3010. 
Handschin C \& Spiegelman BM 2006 Peroxisome proliferator-activated receptor gamma coactivator 1 coactivators, energy homeostasis, and metabolism. Endocrine Reviews 27 728-735.

Hiscock N, Chan MH, Bisucci T, Darby IA \& Febbraio MA 2005 Skeletal myocytes are the source of Interleukin-6 mRNA expression and protein release during contraction: evidence of fiber type specificity. FASEB Journal 18 992-994.

Hotamisligil GS 2006 Inflammation and metabolic disorders. Nature 444 860-867.

Inoue $\mathrm{H}$, Ogawa W, Asakawa A, Okamoto Y, Nishizawa A, Matsumoto M, Teshigawara K, Matsuki Y, Watanabe E, Hiramatsu R et al. 2006 Role of hepatic STAT3 in brain-insulin action on hepatic glucose production. Cell Metabolism 3 267-275.

Kelly M, Keller C, Avilucea PR, Keller P, Luo Z, Xiang X, Giralt M, Hidalgo J, Saha AK, Pedersen BK et al. 2004 AMPK activity is diminished in tissues of IL-6 knockout mice: the effect of exercise. Biochemical and Biophysical Research Communications 320 449-454.

Klover PJ, Zimmers TA, Koniaris LG \& Mooney RA 2003 Chronic exposure to Interleukin-6 causes hepatic insulin resistance in mice. Diabetes 52 2784-2789.

Klover PJ, Clementi AH \& Mooney RA 2005 Interleukin-6 depletion selectively improves hepatic insulin action in obesity. Endocrinology 146 3417-3427.

Kristiansen OP \& Mandrup-Poulsen T 2005 Interleukin-6 and diabetes: the good, the bad, or the indifferent? Diabetes 54 (Suppl 2) S114-S124.

Lazar M 2005 How obesity causes diabetes: not a tall tale. Science 307 373-375.

Mooney RA 2007 Counterpoint: interleukin-6 does not have a beneficial role in insulin sensitivity and glucose homeostasis. Journal of Applied Physiology $102816-818$

Nordfors L, Hoffstedt J, Nyberg B, Thörne A, Arner P, Schalling M \& Lönnqvist F 1998 Reduced gene expression of UCP2 but not UCP3 in skeletal muscle of human obese subjects. Diabetologia 41 935-939.

Pedersen BK \& Febbraio MA 2007 Point: interleukin-6 does have a beneficial role in insulin sensitivity and glucose homeostasis. Journal of Applied Physiology 102 814-816.

Petersen EW, Carey AL, Sacchetti M, Steinberg GR, Macaulay SL, Febbraio MA \& Pedersen BK 2005 Acute IL-6 treatment increases fatty acid turnover in elderly humans in vivo and in tissue culture in vitro. American Journal of Physiology. Endocrinology and Metabolism 288 E155-E162.

Ruderman NB, Keller C, Richard AM, Saha AK, Luo Z, Xiang X, Giralt M, Ritov VB, Menshikova EV, Kelley DE et al. 2006 Interleukin-6 regulation of AMP-activated protein kinase. Potential role in the systemic response to exercise and prevention of the metabolic syndrome. Diabetes $\mathbf{5 5}$ (Suppl 2) S48-S54.

Schrauwen P \& Hesselink M 2002 UCP2 and UCP3 in muscle controlling body metabolism. Journal of Experimental Biology 205 2275-2285.
Senn JJ, Klover PJ, Nowak IA, Zimmers TA, Koniaris LG, Furlanetto RW \& Mooney RA 2003 Suppressor of cytokine signaling-3 (SOCS-3), a potential mediator of interleukin-6-dependent insulin resistance in hepatocytes. Journal of Biological Chemistry 278 13740-13746.

Starkie RL, Rolland J, Angus DJ, Anderson MJ \& Febbraio MA 2001 Circulating monocytes are not the source of elevations in plasma IL-6 and TNF-alpha levels after prolonged running. American Journal of Physiology. Cell Physiology 280 C769-C774.

Starr R, Willson TA, Viney EM, Murray LJ, Rayner JR, Jenkins BJ, Gonda TJ, Alexander WS, Metcalf D, Nicola NA et al. 1997 A family of cytokineinducible inhibitors of signalling. Nature 387 917-921.

Steensberg A, Febbraio MA, Osada T, Schjerling P, van Hall G, Saltin B \& Pedersen BK 2001 Interleukin-6 production in contracting human skeletal muscle is influenced by pre-exercise muscle glycogen content. Journal of Physiology 537 633-639.

Torisu T, Sato N, Yoshiga D, Kobayashi T, Yoshioka T, Mori H, Iida M \& Yoshimura A 2007 The dual function of hepatic SOCS3 in insulin resistance in vivo. Genes to Cells 12 143-154.

Ueki K, Kondo T \& Kahn CR 2004 Suppressor of cytokine signaling 1 (SOCS-1) and SOCS-3 cause insulin resistance through inhibition of tyrosine phosphorylation of insulin receptor substrate proteins by discrete mechanisms. Molecular and Cellular Biology 24 5434-5446.

Vozarova B, Weyer C, Hanson K, Tataranni PA, Bogardus C \& Pratley RE 2001 Circulating interleukin-6 in relation to adiposity, insulin action, and insulin secretion. Obesity Research 9 414-417.

Wallenius V, Wallenius K, Ahren B, Rudling M, Carlsten H, Dickson SL, Ohlsson C \& Jansson JO 2002 Interleukin-6-deficient mice develop mature-onset obesity. Nature Medicine 8 75-79.

Watt MJ, Dzamko N, Thomas W, Rose-John S, Ernst M, Carling D, Kemp BE, Febbraio MA \& Steinberg GR 2006a CNTF reverses obesity-induced insulin resistance by activating skeletal muscle AMPK. Nature Medicine 12 541-548.

Watt MJ, Steinberg GR, Chen Z-P, Lee JS, Kim CK, Kemp BE \& Febbraio MA $2006 b$ Fatty acids stimulate AMP-activated protein kinase and enhance fatty acid oxidation in skeletal muscle. Journal of Physiology 574 139-147.

Wellen KE \& Hotamisligil GS 2005 Inflammation, stress, and diabetes. Journal of Clinical Investigation 115 1111-1119.

Wojtaszewski JF, Hansen BF, Gade J, Kiens B, Markuns JF, Goodyear LJ \& Richter EA 2000 Insulin signaling and insulin sensitivity after exercise in human skeletal muscle. Diabetes 49 325-331.

Received in final form 22 May 2008

Accepted 2 June 2008

Made available online as an Accepted Preprint 3 June 2008 